\title{
Internationale Konferenz über Induratio penis plastica (IPP) 17.-19. März 1993, Washington Bethesda
}

\author{
W. Weidner \\ National Institutes of Health, Bethesda, Devine-Center for Genitourinary Reconstruction, \\ Sentara Norfolk General Hospital, Norfolk
}

Das NIH organisierte in Zusammenarbeit mit dem Devine-Center for Genitourinary Reconstruction in Bethesda vom 17.-19. März 1993 eine Konsensuskonferenz über die Induratio penis plastica (Peyronie's disease). Unter der Leitung von Charles J. Devine (Sentara Norfolk) diskutierten 200 eingeladene Wissenschaftler aus den Vereinigten Staaten, England, Österreich, Schweden und Indien über Ätiologie, Pathogenese, Diagnostik und Therapie dieser in weiten Bereichen immer noch unklaren Erkrankung.

\section{Klassifikation, Ätiologie und Pathogenese}

In seinem Einleitungsreferat wies C. J. Devine auf die Einordnung des Krankheitsbildes in den Formenkreis der Fibromatosen mit enger Assoziation zur Dupuytren'schen Kontraktur und Plantarfibromatose (M. Ledderhose) hin. Seines Erachtens handelt es sich um ein primär entzündliches, dann narbiges Krankheitsbild, das etwa $1 \%$ aller Männer betrifft und bei über $90 \%$ der Patienten zu einer nach dorsal gerichteten Deviation des Penis führt. Die Ätiopathogenese dieses Krankheitsbildes ist bis heute unklar. Die Pathomorphologie ist durch die grundlegenden Untersuchungen von Smith aus dem Jahr 1966 definiert (1). Davis (Washington) unterscheidet entsprechend eine primäre entzündliche Phase mit Infiltration der Tunica albuginea durch Lymphozyten und Plasmazellen und anschließende narbige Phase mit Proliferation von Fibroblasten und terminaler Kalzifikation. Lue (San Francisco) weist darauf hin, daß durch die besondere Anordnung der dorsalen Tunica bedingt, die aus mehr als 20 elastischen Lagen besteht, die dorsale Bevorzugung des Krankheitsbildes zu erklären ist. Da das Corpus spongiosum nach ventral nur eine einschichtige elastische Umhüllung besitzt, kommt ein ventraler Plaques nur in Ausnahmefällen vor.

Devine (Norfolk) und Gelbard (Los Angeles) glauben an die traumatische Genese der IPP durch unterschiedlich starke mechanische Belastung der Tunica albuginea mit primärer Exsudation von Fibrin und konsekutiver überschießender Kollagenbildung. Somers (Cleveland) unterstützt diese Hypothese. Nach seiner Meinung wird die Kollagendeposition bei Induratio penis plastica durch Verschiebungen der unterschiedlichen Kollagenmuster erklärt. Seine Erklärung der primären Noxe ist eine Fibrinexsudation in Gewebespalten mit Aktivierung der Fibrose-

Akt. Urol. 24 (1993) 372-374

(c) Georg Thieme Verlag Stuttgart · New York bildung. Während zytogenetische Faktoren keine Rolle spielen, berichtet Leffell (Baltimore) über eine erhöhte Inzidenz vieler HLA-Gruppen bei Patienten mit IPP. Es sind sowohl Kreuzreaktionen in den Gruppen HLA-B7 und HLA-B27 (Ralph, London), sowie zur HLA-Klasse 2 (Rompel, Gießen) erarbeitet worden. Ralph und Pryor (London) berichten zusätzlich über Antikörperreaktionen gegen bakterielle Antigene als fraglicher Auslöser einer IPP.

Zusammenfassend konnte in diesem ersten Abschnitt der Konferenz kein Konsensus über die Ätiopathogenese des Krankheitsbildes erarbeitet werden. Das erstmals von Lue vorgestellte TIERMODELL könnte hier eine Verbesserung unseres Verständnisses bringen. Die Autoren induzierten durch Injektion von Dihydroxyfumarat in die Tunica albuginea bei Ratten „IPP-ähnliche Veränderungen“. Leider sind die Untersuchungen nicht über einen Zeitraum von mehr als 6 Wochen fortgeführt worden. Dihydroxyfumarat ist eine superoxydfreisetzende Substanz, die über eine Stimulation von Makrophagen und Leukozyten zu einer entzündlichen Reizung und zu einer Erhöhung der Kollagensynthese führen soll.

\section{Klinisches Beschwerdebild und Diagnostik}

In einem zweiten Block wurden der klinische Verlauf, die typische Symptomatik und die optimale Diagnostik diskutiert. Gelbard (Los Angeles) wies darauf hin, daß der tastbare dorsale Plaques Leitsymptom des Krankheitsbildes ist. Nach seinen Erfahrungen bleibt die Deviation in $89 \%$ aller Fälle konstant. Die Schmerzsymptomatik kann variieren. Wagner (Kopenhagen) weist auf die Wichtigkeit des Schwellkörpers und der „venookklusiven Dysfunktion“ (Leakage) als Ursache der erektilen Dysfunktion bei Induratio penis plastica hin. Weidner (Göttingen) findet in einer multizentrischen Untersuchung an 222 Männern in $91 \%$ eine dorsale Deviation, in $71 \%$ Erektionsschmerzen und immerhin bei 56 Männern einen kompletten Erektionsverlust. 47 dieser 56 Patienten zeigen eine pathologische dynamische Infusions-Kavernosographie und -Kavernosometrie (DICC). Ähnliche Befunde werden von Krane (Boston) präsentiert, der bei $91 \%$ der 42 untersuchten impotenten Patienten eine venookklusive Dysfunktion findet, wobei eine plaqueassoziierte Insuffizienz im Vordergrund steht. Gordon (Norfolk) empfiehlt vor jeder chirurgischen Therapie der IPP unabdingbar eine DICC, um die Erektionsfähigkeit ausreichend zu evaluieren. Broderick (Philadelphia) berichtet über Farbduplexsonographie-Erfahrungen an 20 Patienten mit Induratio penis plastica. Er kommt dabei zu dem Schluß, daß eine Minderperfusion der Schwellkörperarterien nicht mit 
dem Krankheitsbild korreliert werden kann. Trotzdem empfiehlt der Autor eine regelmäßige Farbduplexsonographie vor jeder chirurgischen Therapie der Induratio penis plastica, um eine arterielle Minderversorgung der Schwellkörperarterien als Ursache sexueller Funktionsstörung beurteilen zu können.

Die anschließende Diskussion versuchte eine optimierte Diagnostik der IPP festzulegen. Einigkeit bestand darin, bei jedem Patienten eine ausführliche Sexualanamnese und falls möglich, eine Autofotografie nach Kelami durchführen zu lassen. Der Penis sollte bei maximaler Streckung nach dorsal und ventral palpiert werden. Über die Durchführbarkeit einer Weichteil-Röntgenaufnahme konnte keine Einigkeit erzielt werden, da die hohe Strahlenbelastung dieser radiologisch veralteten Methode von den teilnehmenden Radiologen negativ kommentiert wurde. Shabsigh (New York) berichtete über seine Erfahrungen mit der hochauflösenden Ultraschalldiagnostik, wobei er nur in $27 \%$ Verkalkungen bei 48 untersuchten Patienten nachweisen konnte. Er wies darauf hin, daß verdächtige Schatten im Sonogramm histologisch nicht einer morphologisch nachweisbaren Verkalkung entsprechen müssen. Seine Arbeitsgruppe führt die Ultraschalluntersuchung grundsätzlich ohne artifizielle Erektion durch. In der nachfolgenden Diskussion konnte keine Übereinstimmung erzielt werden, ob zur Ultraschalldiagnostik vorweg eine Injektion von vasoaktiven Substanzen durchgeführt werden sollte. Es bestand nur insoweit ein Konsensus, daß eine subtile Ultraschalluntersuchung von der Peniswurzel bis zur Eichel und von dorsal und ventral durchgeführt werden soll. Die Kernspintomographie wurde überraschend im Gegensatz zur Sonographie als wenig hilfreich angesehen (Persky, Tampa).

Krane (Boston) wies abschließend noch einmal darauf hin, daß seines Erachtens die Durchführung einer dynamischen Infusionscavernosographie und Cavernosometrie (DICC) nach den Boston-Kriterien (Erhaltungsfluß, Druckabfall) bei allen Patienten mit Erektionsverlust unabdingbar ist. Diesem Vorschlag schloß sich die Konferenz an.

Als Tip nachzutragen ist, daß für die Verifizierung des Erektionswinkels die fotografische Dokumentation unter einer Vakuumdevice durchgeführt werden kann.

\section{Nicht-operative Therapie}

Die medikamentöse Therapie der Induratio penis plastica konnte nicht einvernehmlich bewertet werden. Es bestand einzig Übereinstimmung, daß mit allen nachfolgend genannten Substanzen prospektive plazebokontrollierte Studien durchgeführt werden sollten. Gelbard (Los Angeles) berichtete über seine Erfahrungen mit der lokalen Injektion von Kollagenase und konnte keine signifikanten Unterschiede zur Plazebo-Gruppe nachweisen. Schellenberg (Norfolk) berichtete über die theoretischen Möglichkeiten der lokalen Therapie mit Superoxiddismutase, klinische Studien $\mathrm{zu}$ dieser in Deutschland häufig eingesetzten Substanz (Orgotein) wurden leider nicht vorgestellt. Devine berichtete über die Vitamin-E-Strategie, die nach seiner Meinung in Ein- zelfällen wirksam ist. Carson (Durham) berichtete über Ansätze mit Paraminobenzoat (Potaba ${ }^{\circledR}$ ), wobei in einer offenen prospektiven Studie bei 20 von 36 Patienten der Schmerz, bei 24 dieser Fälle der Plaques und bei 30 der Patienten die Kurvatur gebessert wurde. Er ist der Meinung, daß die Substanz über eine verminderte Glukosaminproduktion wirkt und ein minimales Therapieintervall von drei Monaten eingehalten werden sollte. Levine (Chicago) berichtete über die ersten Erfahrungen mit der intraläsionalen Injektion von Verapamil, was durch eine Hemmung der Inkorporation von Prolin in die Kollagenstruktur die Kollagensynthese behindern soll. Er berichtete in einer Pilotstudie an 14 Männern über eine Verbesserung der Schmerzen in 10 Fällen. Weiter wurde auf die eventuelle Wirksamkeit von Colchizin (Lue), Interferon (Gelbard) und Tamoxifen (Ralph) hingewiesen. Tamoxifen hat sich bereits bei der retroperitonealen Fibrose als wirksam erwiesen und supprimiert in einer Dosis von $20 \mathrm{mg} / \mathrm{Tag}$ die Aktivität von Makrophagen und Fibroblasten. Abgeschlossen wurde die konservative Therapie durch ein Übersichtsreferat von Carson, der über die niedrig dosierte Radiatio der Induratio penis plastica berichtete. Nach seinen Erfahrungen sollte diese maximal mit 400-600 rad durchgeführt werden, wobei er den Linearbeschleuniger einsetzt. Er hat bisher nur ein Ansprechen des Symptoms „Schmerz" verifizieren können.

Grundsätzlich bestand Übereinstimmung, daß in der Entzündungsphase der Induratio penis plastica (Aktivierungsphase) ein medikamentöses Intervall jeder chirurgischen Therapie vorgeschaltet werden sollte, wobei jede Schmerzsymptomatik als Charakteristikum des "Nicht-Endstadiums“ einer IPP anzusehen ist (Devine, Lue, Ralph, Austoni).

\section{Chirurgische Therapie}

Bei der chirurgischen Therapie der IPP muß nach Krane grundsätzlich zwischen Patienten mit und ohne erektiler Dysfunktion unterschieden werden. Jordan (Norfolk) gab eine Übersicht über die chirurgischen Therapiemöglichkeiten, wobei die Nesbit-Operation, die Inzision und Exzision der Plaques mit und ohne Deckung mit Goretex, Dermal-Graft, Tunica vaginalis, Temporalisfaszie, Venen sowie die Implantation einer Penisprothese diskutiert wurden.

Übereinstimmung bestand, daß eine schmerzhafte IPP als chirurgische Kontraindikation anzusehen ist.

Bei erhaltener Erektion und vorhandener Deviation favorisieren Ralph und Pryor (London) die Nesbit-Technik, wobei $1 \mathrm{~mm}$ Exzision der Tunica mit 10 ${ }^{\circ}$-Korrektur des Deviationswinkels gleichzusetzen ist. Die Autoren verwenden grundsätzlich 0er PDS mit invertierter Stichrichtung, um eine im Gewebe geborgene Knotenbildung zu erreichen. Sie berichteten bei 359 operierten $\mathrm{Pa}$ tienten nur in 17 Fällen über eine den Koitus behindernde Penisverkürzung. Dieser Feststellung wurde von der Norfolk-Arbeitsgruppe (Jordan, Devine) scharf widersprochen, die darauf hinwiesen, daß zumindest in den Vereinigten Staaten kein Patient sich freiwillig auf eine Verkür- 
zung des Penis durch eine derartige Technik einlassen würde.

Leider fehlten in diesem Zusammenhang Ergebnisse der Penisraffplastik, die nach Ansicht des Berichterstatters identische Ergebnisse zur Nesbittechnik bringt.

Anschließend berichteten unterschiedliche Autoren über die Behandlung der Deviation durch Inzision oder Exzision der Plaques und anschließendes Grafting. Dabei wiesen Schlossberg und Jordan (Norfolk) darauf hin, daß beim dorsalen Plaques immer die Vena dorsalis penis profunda mitentfernt werden sollte. Beide Autoren stellten noch einmal ausführlich die DermalGraft-Technik vor und unterstrichen, daß nach ihrer Meinung nur $10 \%$ aller vor der Operation voll erektionsfähigen IPP-Patienten postoperativ eine venöse Insuffizienz und erektile Dysfunktion entwickeln würden. Auffällig war, daß die Norfolk-Gruppe trotz ihrer sehr großen Erfahrungen mit der Dermal-Graft-Technik nicht über Langzeitergebnisse berichtete. Das (Walnut Creek) berichtete über seine Erfahrungen mit Tunica-vaginalis-Grafting, wobei er einen Verlauf von 96 Monaten überblickte und zu insgesamt guten Erfolgen kam, die insbesondere bei kleineren Plaques $(<2 \mathrm{~cm})$ die Verwendung dieses Materials empfehlen lassen. Gelbard verwendet für das Grafting die Faszia temporalis, wobei er in $13 \%$ ein "Penisshortening" bemerkte. Lue verwendet bei Patienten ohne Erektionsstörungen mit Deviation nur die Nesbit-Operation, neuerdings aber auch auf der Seite der Kurvatur eine Inzisionstechnik unter Belassung der Plaques, die er dann nach einer früher von Gelbard angegebenen Methode mit Vena dorsalis profunda mit dem Endothel zum Schwellkörper gerichtet abdeckt. Dabei wird der Plaques nur inzidiert, niemals exzidiert. In ähnlicher Form geht Gelbard vor, der unter Belassung der Plaques durch Inzisionen der kontraktierten Region mit nachfolgender Implantation von Temporalisfaszie eine Begradigung vornimmt. Hellstrom (New Orleans) verwendet bei gleicher Technik nur Goretex. Lue führt alle Eingriffe unter künstlicher Erektion durch. Er setzt in Einzelfällen auch eine Laserevaporisation von verkalkten IPP-Herden ein.

Austoni (Mailand) berichtet abschließend über seine Erfahrungen an 335 Patienten, wobei er nach einer vollständigen Separation von dorsalen Gefäßnervenbündeln und Schwellkörpern eine Exzision der Plaques und Rekonstruktion mit Dermal-Graft durchführte. Er kam bei seinem großen Patientengut, das leider nicht sehr detailliert analysiert war, zu einer postoperativen Impotenzrate von $10 \%$. Bis heute sind dies jedoch die einzigen Langzeituntersuchungen nach Plaques-Exzision und Deckung.

Abschließend wurde die Frage diskutiert, ob bei Deckung entsprechender Resektionsstellen mit Dermal-Graft Vorteile gegenüber Goretex, TemporalisFaszie, Tunica vaginalis und Vena-penis-profunda-Material bestehen. Der Vorteil des erstgenannten Materials wurde von der Norfolk-Gruppe stark propagiert, die nach ihren Untersuchungen davon ausgeht, daß das DermalGraft nach einigen Monaten in Kollagen und elastische Fasern umgewandelt ist. Hier konnte kein Konsensus er- zielt werden; viele Teilnehmer schlossen sich der Meinung der Boston-Arbeitsgruppe (Krane, Vickers) an, daß die hohe Rate postoperativer Impotenzen gerade die DermalGraft-Technik belastet. Einigkeit bestand nur darin, daß vor jeder chirurgischen Therapie 1. keine Schmerzhaftigkeit bestehen sollte, und 2. durch DICC eine Schwellkörperinsuffizienz sicher ausgeschlossen werden muß.

Bei vorhandener erektiler Dysfunktion wird immer eine Penisprothese implantiert.

\section{Prothesenchirurgie}

Montague (Cleveland) berichtete über 61 Patienten mit IPP und Impotenz, die er mit verschiedenen hydraulischen Prothesen-Typen therapierte und bei denen er bei Deviation in 30\% ein "Modelling" durchführte. Er empfahl eine Inzision über der Kurvatur über liegender Prothese ohne entsprechende Deckung. Nur 14\% der Prothesen mußten explantiert werden. Er wies in der Diskussion auch auf das von Wilson (Arkansas) empfohlene "Peniscracking" hin, wobei bei implantierter und insufflierter Prothese der "Plaques" sozusagen gebrochen und der Penis dann auf der Prothese modelliert wird.

\section{Allgemeine Anmerkungen}

Abschließend beschäftigten sich Jordan und Devine (Norfolk) mit den Komplikationen der Plaques-Chirurgie und wiesen darauf hin, daß bei jeder Urethralverletzung die Operation beendet werden muß. Hämatome sollten wegen der Induktion einer Penisverkürzung und gehäuften Glanshypoästhesien sofort nachoperiert werden. Um entsprechende Störungen frühzeitig zu vermeiden, empfiehlt Devine grundsätzlich die Anwendung eines postoperativen bioocclusiven Dressings, was durch seine Transparenz jede Form einer Gewebeschädigung erkennen läßt.

Zusammenfassend ergab die Konferenz ein Update moderner diagnostischer und therapeutischer Verfahren der Induratio penis plastica. Vermißt wurde vom Berichterstatter jeder Versuch, die chirurgische Therapie zu standardisieren. Vielleicht ist auch die Zeit hierfür noch zu früh.

\section{Literatur}

1 Smith, B. H.: Peyronie's disease. Amer. J. Clin. Pathol. 45 (1966) 670

Prof. Dr. W. Weidner

Direktor der Urologischen Klinik

Justus-Liebig-Universität

Klinikstr. 29

35392 Gießen 